[0212-7199 (2008) 25: 3; pp 131-133] ANALES DE MEDICINA INTERNA Copyright (C) 2008 ARAN EDICIONES, S.L.

AN. MED. InTERna (Madrid) Vol. 25, N. ${ }^{\circ} 3$, pp. 131-133, 2008

\title{
Insuficiencia suprarrenal causada por un linfoma no- Hodgking B primario suprarrenal: presentación de un caso y revisión de la literatura
}

\author{
B. HERNÁNDEZ MARÍN, V. M. DÍAZ MUÑOZ DE LA ESPADA, R. ÁLVAREZ \\ ÁLVAREZ, S. ENCINAS GARCÍA, P. KHOSRAVI SHAHI, R. PÉREZ FERNÁNDEZ, \\ G. PÉREZ MANGA
}

Servicio de Oncología Médica. Hospital General Universitario Gregorio Marañón. Madrid

\begin{abstract}
ADRENAL FAILLURE CAUSED BY PRIMARY ADRENAL NONHODGKING LYMPHOMA: A CASE REPORT AND REVIEW OF THE LITERATURE
\end{abstract}

\section{RESUMEN}

Presentamos el caso de un varón de 78 años que ingresa por un cuadro de debut de insuficiencia suprarrenal. Se realizó un estudio TC que mostró masas suprarrenales bilaterales de hasta $10 \mathrm{~cm}$. Se completó estudio con biopsia percutanea de masa suprarrenal y biopsia de médula ósea, siendo diagnosticado de Linfoma no Hodgkin B difuso de células grandes primario suprarrenal con afectación suprarrenal bilateral. El paciente fue tratado con quimioterapia según esquema R-CHOP (Rituximab, Ciclofosfamida, Doxorrubicina liposomal, Vincristina y Prednisona). Tras 4 ciclos de quimioterapia se objetivo una respuesta parcial radiológica. Se suspendió la quimioterapia por toxicidad grado IV, completándose el tratamiento con RT sobre masa suprarrenal derecha. El paciente falleció por cuadro séptico pocos días después de finalizar la radioterapia, sin objetivarse progresión de la enfermedad.

PALABRAS CLAVE: Linfoma suprarrenal. Insuficiencia suprarrenal. Enfermedad de Addison.

\section{ABSTRACT}

We report a case of 78-year old man who presented with symptoms of adrenal insufficiency. The computed tomography (CT) scan showed the presence of bilateral adrenal masses. A CT-scan guided needle biopsy revealed diffuse large- $B$ cell lymphoma. The absence of pathological findings in clinical, bone marrow and CT scan examinations supported the diagnosis of primary non-Hodgkin Lymphoma of the adrenal glands. The patient was treated with four cycles of $R$-CHOP chemotherapy with Rituximab, liposomal Doxorubicin, Cyclophosphamide, Vincristine and Prednisolone. At the end of fourth cycle there was radiological improvement but the chemotherapy was stopped because of IV grade toxicity. He completed treatment with radiotherapy of right adrenal mass. Few days after finishing radiation therapy the pacient died due to a disseminated infection. No progressive disease was founded.

KEY WORDS: Adrenal lymphoma. Adrenal faillure. Addison disease.

Hernández Marín B, Díaz Muñoz de la Espada VM, Álvarez Álvarez R, Encinas García S, Khosravi Shahi P, Pérez Fernández R, PérezManga G. Insuficiencia suprarrenal causada por un linfoma no-Hodgking B primario suprarrenal: presentación de un caso y revisión de la literatura. An Med Interna (Madrid) 2008; 25: 131-133.

\section{INTRODUCCIÓN}

El linfoma suprarrenal primario (LSP) constituye menos del $1 \%$ de los linfomas extraganglionares. Esta infrecuente entidad se asocia en algunos casos a la aparición de un cuadro de insuficiencia suprarrenal secundario a la infiltración tumoral de las glándulas adrenales. Esta situación suele ocurrir cuando la afectación neoplásica es bilateral. La mayoría de los LSP son linfomas B de alto grado, generalmente del subtipo de células grandes. A diferencia de otros linfomas B de alto grado, el pronóstico de los pacientes con LSP es muy pobre, con una mediana de supervivencia desde el diagnóstico menor a un año. Presentamos el caso de un paciente con un cuadro de insuficiencia suprarrenal aguda secundario a un LSP con afectación bilateral. Además realizamos una revisión de la literatura sobre las características clinicopatológicas mas relevantes del LSP.

\section{CASO APORTADO}

Un varón de 78 años ingresó en nuestro centro con un cuadro clínico consistente en deterioro del estado general, vómitos intensos, hipotensión, taquicardia y dolor abdominal. No presentaba síndrome febril. Su único antecedente médico de interés era un infarto agudo de miocardio anterolateral 15 años antes, sin secuelas sobre la fracción de eyección. La exploración física no mostró hallazgos patológicos significativos. El hemograma y la bioquímica sanguineas revelaron como datos e interés: Hb $11 \mathrm{~g} / \mathrm{dl}, \mathrm{LDH} 683 \mathrm{UI} / \mathrm{l}, \mathrm{Na} 125$ $\mathrm{mmol} / \mathrm{l}, \mathrm{K} 5,3 \mathrm{mmol} / \mathrm{l}, \mathrm{Cl} 94 \mathrm{mmol} / \mathrm{l}$, urea $40 \mathrm{mg} / \mathrm{dl}, \mathrm{Cr}$ 0,9 mg/dl. El resto de parámetros no presentaba alteraciones. La persistencia de hipotensión a pesar de las medidas de soporte iniciales, junto con los hallazgos analíticos sugería un posible cuadro de insuficiencia suprarrenal. El nivel de cortisol basal sérico a las 8:00 a.m. fue de 4,8 $\mu \mathrm{g} / \mathrm{dl}$ y tras la estimulación con 250 microgramos intravenosos de ACTH solo aumentó a 5,7 $\mu \mathrm{g} / \mathrm{dl}$. Tras confirmar el diagnóstico de 
insuficiencia suprarrenal se inició el tratamiento sustitutivo corticoideo, consiguiendo la estabilización del cuadro. El estudio TC reveló masas suprarrenales derecha e izquierda de $10 \mathrm{~cm}$ y $7,5 \mathrm{~cm}$ respectivamente (Fig. 1). No se objetivaron adenopatías de tamaño patológico, esplenomegalia ni alteraciones radiológicas en otros órganos. Los niveles de adrenalina, noradrenalina y dopamina séricos, así como los de ácido vanilmandélico en diuresis de 24 horas, estaban en rango normal. Debido a la alta sospecha de infiltración suprarrenal de origen neoplásico, se realizó una biopsia percutanea guiada por TC. La anatomía patológica demostró una infiltración difusa por células linfoides atípicas de gran tamaño, cuyo inmunofenotipo presentaba positividad para CD45 (antígeno común leucocitario), CD20, CD79a, CD43, Bcl-2 e inmunoglobulina de superficie, siendo negativo para CD3, CD5, CD30, CD38, CD10, CD23, CD43 y bcl-6. Esta histología era compatible con una infiltración de la glándula suprarrenal por un linfoma B difuso de células grandes.

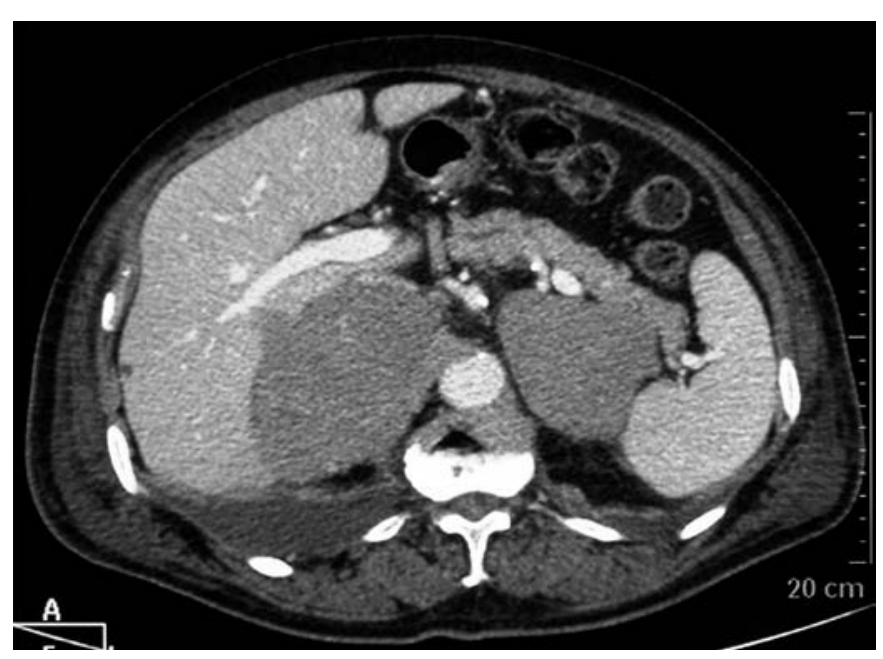

Fig. 1.

Se completó el estudio de extensión con una biopsia de médula ósea, que no mostró infiltración por el linfoma. Ante estos hallazgos se llegó al diagnóstico de linfoma no-Hodgkin $\mathrm{B}$ difuso de células grandes primario suprarrenal con afectación bilateral.

El paciente fué tratado con poliquimioterapia según esquema Rituximab-CHOP trisemanal (Rituximab $375 \mathrm{mg} / \mathrm{m}^{2}$ día 1, Ciclofosfamida $750 \mathrm{mg} / \mathrm{m}^{2}$ día 1, Doxorrubicina liposomal $50 \mathrm{mg} / \mathrm{m}^{2}$ día 1 , Vincristina $1,4 \mathrm{mg} / \mathrm{m}^{2}$ día 1 , Prednisona $100 \mathrm{mg} / \mathrm{m}^{2} 5$ días consecutivos). Se decidió sustituir la adriamicina por doxorrubicina liposomal, para disminuir el riesgo de cardiotoxicidad, debido a los antecedentes cardiológicos del paciente. El paciente recibió 4 ciclos de quimioterapia, tras los cuales se objetivó una respuesta parcial radiológica, con una reducción del tamaño de la masa suprarrenal derecha mayor al 50\%, y desaparición de la masa suprarrenal izquierda (Fig. 2). La aparición de un exceso de toxicidad grado IV (incluyendo sepsis por neutropenia severa y descompensación de su insuficiencia suprarrenal) así como un episodio de cardiopatía isquémica, impidió continuar con el tratamiento de quimioterapia. Se decidió completar el tratamiento con radioterapia (36 Gy) sobre la masa suprarrenal derecha persistente. Pocos días después de finalizar el tratamiento con radioterapia, el paciente falleció por un cuadro séptico sin objetivarse progresión de su enfermedad oncológica. No se realizó autopsia.

\section{DISCUSIÓN}

La infiltración de la glándula suprarrenal en los linfomas no-Hodgkin puede aparecer hasta en un $25 \%$ de los casos (1).

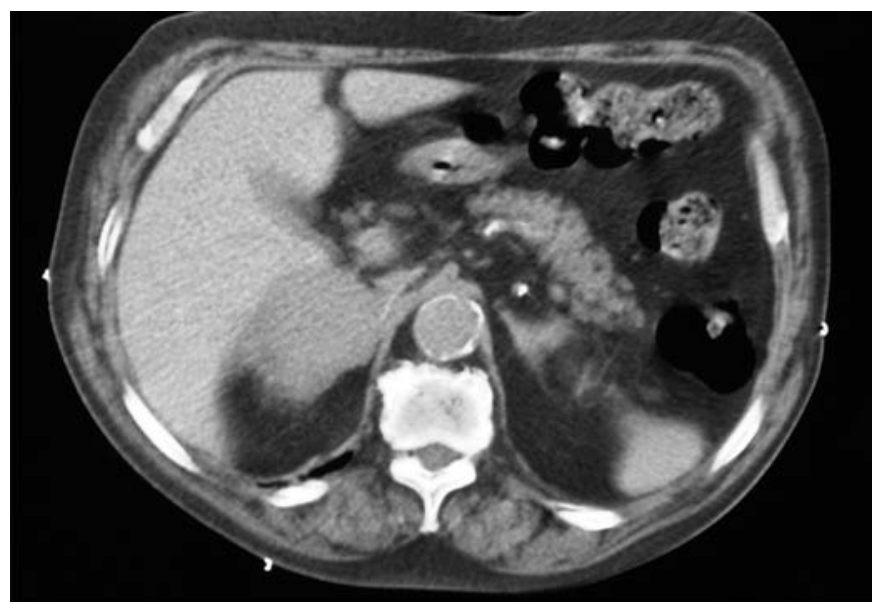

Fig. 2.

La afectación suele ser unilateral y generalmente se trata de pacientes en estadios avanzados.

Los linfomas extraganglionares (LE) constituyen aproximadamente el $30 \%$ de los linfomas no-Hodgkin (LNH) (2). Se han descrito casos de LE en casi todos los órganos, aunque la incidencia de aparición en cada localización es muy diferente (3). En general, el tratamiento de los LNH extraganglionares debe abordarse con intención curativa. La mayoría de los LE se presentan localizados y por tanto el tratamiento local (cirugía o radioterapia) tiene un papel importante en su tratamiento. Sin embargo, el elevado número de recaídas cuando solo se aplica tratamiento local, hace recomendable el uso de quimioterapia sistémica como parte del tratamiento inicial de estos pacientes.

El linfoma primario de la glándula suprarrenal representa alrededor del $1 \%$ de los linfomas extraganglionares y se han publicado menos de 100 casos en la literatura (2). Es más frecuente en hombres que en mujeres $(2 / 1)$ y la media de edad se sitúa en torno a los 68 años (4). En el $65 \%$ de los casos la afectación suprarrenal es bilateral (5). El $90 \%$ de los LSP son linfomas No-Hodgkin B de células grandes (6), aunque en la literatura se describen algunos casos de linfomas $\mathrm{T}$ (7) y linfomas NK (8).

Los síntomas más frecuentes de presentación son dolor abdominal, dolor lumbar, fiebre y pérdida de peso (9). Cuando la afectación suprarrenal es unilateral no es frecuente la aparición de insuficiencia suprarrenal. Sin embargo, en los pacientes con afectación bilateral puede objetivarse algún grado de insuficiencia suprarrenal asociada hasta en el $60 \%$ de los casos, aunque suele ser subclínica en la mayoría de ellos (10).

A pesar de su baja incidencia, el LSP debe incluirse en el diagnóstico diferencial de una masa suprarrenal. En los estudios TC suele presentarse como masas suprarrenales de gran tamaño, heterogéneas, con signos de necrosis o de hemorragia $(11,12)$. El FDG-PET puede ayudar a determinar la etiología maligna de las masas adrenales (13).

El diagnóstico diferencial incluye distintas entidades. Algunas neoplasias malignas como el carcinoma de mama, pulmón, tumores del tracto gastrointestinal y el melanoma maligno presentan una alta incidencia de metástasis suprarrenales, a menudo bilaterales. En el diagnóstico diferencial también deben incluirse otras entidades como el carcinoma suprarrenal primario, el feocromocitoma y algunos procesos infecciosos. La rea- 
lización de una PAAF presenta una sensibilidad del 50-60\% para el diagnóstico del LSP (14). Sin embargo, en muchos casos para llegar al diagnóstico definitivo se requiere la realización de una biopsia, e incluso una adrenalectomía (15).

El SLP es una entidad con peor pronóstico que otros linfomas B extraganglionares. El $90 \%$ de los casos publicados fallecieron en menos de un año desde el diagnóstico a pesar del tratamiento (16), aunque se han descrito algunos casos de supervivientes a largo plazo (17). EL LSP, en su evolución, presenta una alta incidencia de afectación de múltiples órganos extraganglionares, como el estómago y el SNC (18). Algunos autores sugieren que la asociación de insuficiencia suprarrenal puede implicar un peor pronóstico $(19,20)$. Otros factores de mal pronóstico como son la edad avanzada, el volumen tumoral, la afectación de otros órganos y la elevación de la LDH (21). Las principales causas de muerte son la progresión de la enfermedad, las infecciones severas y el tromboembolismo pulmonar $(3,22)$. En nuestro paciente, un cuadro de septicemia severa, sin evidencia de progresión tumoral, fue la causa de su fallecimiento.

Los esquemas de quimioterapia habitualmente empleados con intención curativa en el tratamiento de los linfomas
B de alto grado, ofrecen resultados muy pobres en los LSP. El tratamiento óptimo del LSP aún no se ha establecido y su escasa incidencia impide la realización de estudios orientados a resolver esta cuestión. La quimioterapia puede obtener buenas respuestas iniciales (23), aunque generalmente son de corta duración y el paciente suele fallecer en menos de 1 año desde el diagnóstico (24). Algunos autores sugieren que la adrenalectomía seguida de quimioterapia podría mejorar los resultados, aunque no existen estudios comparativos que verifiquen esta hipótesis (25). La radioterapia puede mejorar el control local de la enfermedad, pero se desconoce su impacto en la supervivencia de estos pacientes $(3,26)$. Finalmente, la terapia corticoidea de sustitución debe formar parte del tratamiento cuando existe algún grado de insuficiencia suprarrenal.

En el caso que presentamos, el paciente recibió quimioterapia habitual para los linfomas $\mathrm{B}$ de alto grado (R-CHOP) obteniendo una buena respuesta inicial. Se decidió completar el tratamiento con Radioterapia sobre la masa residual. A pesar de que en su evolución no se llegó a objetivar progresión del linfoma, el paciente falleció por un cuadro séptico 9 meses después del diagnóstico.

\section{Bibliografía}

1. Rosenberg SA, Diamond HD, Jaslowitz B, Craver LF. Lymphosarcoma: A review of 1269 cases. Medicine 1961; 40: 31 .

2. Freeman C, Berg JW, Cutler SJ. Ocurrence and prognosis of extranodal lymphomas. Cancer 1972; 29: 252-60.

3. Zucca E, Roggero E, Bertoni F, Cavalli F. Primary extranodal nonHodgkin's lymphomas. Part 1: Gastrointestinal, cutaneous and genitourinary lymphomas. Ann Oncol 1997; 727-37.

4. Wang J, Sun N, Renslo R, Chiang C, Tabarra HJ, Barajas L, et al. Clinically silent primary adrenal lymphoma: A case Report and review of the literature. Am J Hematol 1998; 58: 130-6.

5. Bauduer F, Delmer A, Tourneau AL, Guettier C, Alexandre JH, Zittoun R, Bernadou A. Primary adrenal lymphoma. Acta Haematol 1992; 88: 213-5.

6. Pimentel M, Jonson JB, Allan DR, Greenberg H, Bernstein CN. Primary adrenal lymphoma associated with adrenal insufficiency: A distinct clinical entity. Leuk Lymphoma 1997; 24: 363-7.

7. Schnitzer B, Smid D, Lioyd RV. Primary T-cell lymphoma of the adrenal gland with adrenal insufficiency. Hum Pathol 1986; 17: 634

8. Thompson MA, Habra MA, Routbort MJ, Holsinger FC, Terrier ND, Waguespack SG et al. Primary adrenal natural killer/t-cell nasal type lymphoma: First case report in adults. Am J Hematol; 2006.

9. Hsu CW, Ho CL, Sheu WH, Harn HJ, Chao TY. Adrenal insufficiency caused by primary aggressive non-Hodgkin's lymphoma of bilateral adrenal glands:report of a case and literature review. Ann Hematol 1999; 78: 151-4.

10. Choi CH, Durishin M, Garbadawala ST, Richard J. Non-Hodgkin's lymphoma of the adrenal gland. Arch Pathol Lab Med 1990; 114: 883-5.

11. Alvarez-Castells A, Pedraza S, Tallada N, Castella E, Gifre L, Torrents C. CT of primary bilateral adrenal lymphoma. J Comput Assist Tomogr 1993; 17: 408-9.

12. Salvatore JR, Ross RS. Primary Bilateral Adrenal Lymphoma. Leuk Lymphoma 1999; 34: 111-7.

13. Kumar R, Xiu Y, Mavi A, El-Haddad G, Zhuang H, Alavi A. FDG-PET imaging in primary bilateral lymphoma: A case report and a review of the literature. Clin Nucl Med 2005; 30: 222-30.

14. Dunphy $\mathrm{CH}, \mathrm{Ramos}$ R. Combining fine-needle aspiration and flow cytometric inmunophenotyping in evaluation of nodal and extranodal sites for possible lymphoma: A retrospective review. Diagn Cytopathol 1997; 16: 200-6.

15. Serrano S, Tejedor L, García B, Hallal H, Polo JA, Alguacil G. Addisonian crisis as the presenting feature of bilateral primary adrenal lymphoma. Cancer 1993; 71: 4030-3

16. May F, Bachor R, Hack M, Gottfried H-W, Hautmann RE. Primary adrenal non-Hodgkin's lymphoma: long-term survival. J Urol 1998; 160: 487.

17. Wu HC, Shih LY, Chen TC, Chu SH, Tsai CC. Apatient with bilateral primary lymphoma, presenting with fever of unknown origin and achieving long-term disease-free survival after resection and chemotherapy. Ann Hematol 1999; 78: 289-92.

18. Hahn JS, Choi HS, Suh CO, Lee WJ. A case of primary bilateral adrenal lymphoma (PAL) with central nervous system (CNS) involvement. Yonsei Med J 2002; 43: 385-90.

19. Maugendre D, Derrien C, Grulois I, Simon JP, Guilhem I, Poirier JY et al. Primary adrenal literature with latent adrenal insufficiency: A case report and literature review. Ann Endocrinol 1998; 59: 34-9.

20. Alama MA, Robles A, Roca I, Sales MA, Navarro MC, Roman P. Bilateral primary adrenal lymphoma:an inusual presentation. An Med Interna (Madrid) 2002; 19 (10): 524-6.

21. Gamelin E, Beldent V, Rousselet MC, Rieux D, Rohmer V, Ifrah N Non-Hodgkin's lymphoma presenting with primary adrenal insufficiency: a disease with an underestimated frequency? Cancer 1992; 69: 2333-6.

22. Mantzios G, Tsirigotis P, Veliou F, Boutsikakis I, Petraki L, Kolovos J, et al. Primary adrenal lymphoma presenting as Addison's disease: Case report and review of the literature. Ann Hematol 2004; 83: 460-3.

23. Kuyama A, Takeuchi M, Munemasa M, et al. "Successful tratment of primary adrenal non-hodgkin's lymphoma associated with adrenal insufficiency", Leuk. Lymphoma 2000;38: 203-5.

24. Singh D, Kumar L, Sharma A, Vijayaraghavan M, Thulkar S, Tandon N. Adrenal involvement in non-Hodgkin's lymphoma: Four cases and review of literature. Leuk Lymphoma 2004; 45: 789-94.

25. Utsunomiya $\mathbf{M}$, Takatera $\mathrm{H}$, Itoh $\mathrm{H}$, Tsujimura $\mathrm{T}$, Itatani $\mathrm{H}$. Bilateral primary non-Hodgkin's lymphoma of the adrenal glands with adrenal insufficiency: A case report. Acta Urol Jpn 1992; 38: 311-4.

26. Sasagawa I, Sadamori N, Itoyama T, Tsukasaki K, Nakamura H, Tomonaga M, et al. Primary Adrenal Lymphoma with Chromosomal Abnormalities. Acta Haematol 1995; 94: 156-62. 